\title{
INFRARED ARRAYS AT THE EUROPEAN SOUTHERN OBSERVATORY
}

\author{
G. Finger, G. Nicolini, P. Biereichel, M. Meyer and A. F. M. Moorwood \\ European Southern Observatory
}

\begin{abstract}
This paper gives an overview of infrared array detectors which have been tested and used at ESO. The performance of arrays using Reticon type readouts, CCD readouts and switched FET multiplexers have been evaluated for both $\mathrm{InSb}$ and $\mathrm{Hg}_{1-\mathrm{x}} \mathrm{Cd}_{\mathrm{x}} \mathrm{Te}$ detectors. Performance limitations specific to the NICMOS3 $256 \times 256 \mathrm{Hg}_{1-\mathrm{x}} \mathrm{Cd}_{\mathrm{x}} \mathrm{Te}$ detector installed in the ESO infrared array camera IRAC2 are addressed. The first test results with a high well capacity SBRC 256 x $256 \mathrm{InSb}$ array are also presented.
\end{abstract}

Advanced readout techniques for image sharpening tested on a 2.2-m telescope are discussed briefly. A new generation of instruments being built for the VLT, the very large telescope project of ESO, is designed to house large format 1024 x 1024 IR arrays. A fast data acquisition system is currently being developed at ESO. The system is capable of handling the high data rates generated in the thermal infrared by large format low well capacity arrays. It can also cope with the low read noise required for flux levels of $\leq$ one photon/sec. It will first be installed in ISAAC, the Infrared Array Camera and Spectrometer built for the VLT (Moorwood 1993). The present status of both the detector developments and the data acquisition system is reviewed.

\section{INTRODUCTION}

In less than ten years infrared arrays evolved from formats of $1 \times 32$ to $256 \times 256$ pixels, and their noise performance improved by more than an order of magnitude. In the near future arrays with formats of 1024 x 1024 pixels will be available. A prototype Rockwell 1024 x 1024 $\mathrm{Hg}_{1-\mathrm{x}} \mathrm{Cd}_{\mathrm{x}} \mathrm{Te}$ array has already been used to successfully image the SL-9/Jupiter collision in July 1994. In view of this rapid development it is useful to recapitulate the operating principle and the fundamental performance limit of these devices.

\section{FUNDAMENTAL PERFORMANCE LIMITS OF PHOTOVOLTAIC DETECTORS OPERATING IN CDM}

In the spectral range $\lambda=1$ to $5 \mu \mathrm{m}$ infrared arrays are intrinsic photovoltaic devices operating in the capacitive discharge mode (CDM). The absorbed photon excites an electron from the valence band to the conduction band. If the free electron-hole pair is created within a distance of the pn-junction equal to the diffusion length of the minority carriers it is separated by the electric field of the junction. As shown in Fig. 1 the reset switch connects the diode to the external bias voltage. After this switch is opened the detector junction is floating. Each absorbed photon discharges the capacity by one electron. The integrating node capacity $\mathrm{C}$ is the combined capacity of the detector and the gate of the source follower belonging to each pixel. The integration ramp is sampled at the beginning and end of the exposure. The variance 
of this voltage difference $\{D V\}^{2}$ is given in equation 1 :

$$
\left\{\Delta V(\tau)^{2}\right\}=\frac{2 K T}{C}\left(1-e^{-\frac{\tau}{\tau_{C}}}\right)
$$

$\tau \mathrm{C}$ is the autocorrelation time $\mathrm{RC}$ corresponding to the diode impedance $\mathrm{R}$ and the capacity $\mathrm{C}$ of the integrating node

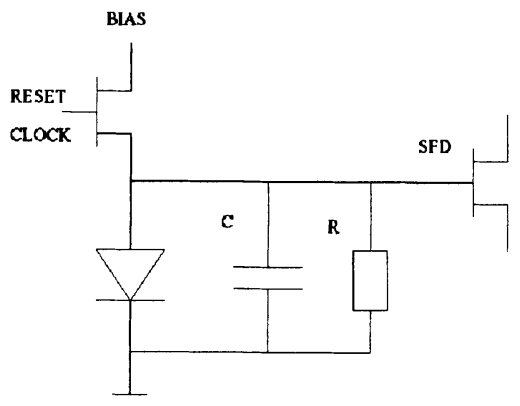

Fig. 1. Photovoltaic detector operating in capacitive discharge mode.

and $\tau$ is the time interval between the two voltage readings. By multiplication with $\mathrm{C}^{2}$ and taking the square root of both sides of equation we obtain the noise $\Delta N r m s(\tau)$ expressed in rms noise electrons as given in equation 2:

$$
\Delta \operatorname{Nrms}(\tau)=\sqrt{\frac{2 K T C}{q^{2}}\left(1-e^{-\frac{\tau}{\tau_{C}}}\right)}
$$

Two limiting cases are of interest assuming typical parameters for state of the art detectors e.g. $\mathrm{C}=30 \mathrm{fF}$, darkcurrent of one $\mathrm{e} / \mathrm{sec}$ at one $\mathrm{V}$ reverse bias, $\tau=600 \mathrm{sec}$ yielding $\tau_{\mathrm{C}}=1.8$ $10^{5} \mathrm{sec}$. For the uncorrelated case, when $\tau>>\tau_{\mathrm{C}}$, equation 2 simplifies to the well known KTC noise $\sqrt{ } 2 K T C / \mathrm{q}$ and yields $51 \mathrm{e}^{-} \mathrm{rms}$. For the correlated case, when $\tau<<\tau_{\mathrm{C}}$, the two voltage readings are completely correlated and equation 2 reduces to $\sqrt{2 K T C \tau} / R / \mathrm{q}$ yielding 2.9 $\mathrm{e}^{-} \mathrm{rms}$. The photon shot noise of photon flux $\Phi_{\text {phot }}$ is equal to $\sqrt{\Phi}_{\text {phot }} \tau$. If the smallest detectable photon flux $\Phi_{\text {limit }}$ is defined to have an associated photon shot noise equal to the correlated case of equation 2, we obtain:

$$
\Phi_{\text {limit }}=\frac{2 K T}{q^{2} R}=0.014 \text { photons } / \mathrm{sec}
$$

For the idealized case of a noise free detector multiplexer and acquisition system the smallest detectable photon flux $\Phi_{\text {limit }}$ is proportional to the detector temperature T and inversely 
proportional to the detector impedance $\mathrm{R}$ and becomes independent of the integration time $\tau$. The HAWAII array (see Table 4) achieves read noise values of $8.6 \mathrm{e} \mathrm{rms}$ which is of the same order of magnitude as the fundamental noise limit derived in equation 3 for the correlated case and long integration times.

The pixel transfer function which gives the signal in volts per electron is inversely proportional to the pixel capacity. To reduce the read noise, the capacity of the detector must be as small as possible. However, in many applications the detector has to handle the high flux levels of the thermal infrared as well. The available readout time depends on the full well of the detector and is proportional to the pixel capacity. To reduce the required read speed for thermal broad band imaging, a trade-off between full well capacity and readout noise has to be considered.

Detectors operating in CDM mode are intrinsically nonlinear devices for two reasons. First, the pixel capacity is a function of voltage which is changing continuously during the integration, since the detector voltage is floating in CDM operation. Second, for small flux levels, the nonlinear I-V characteristic of the diode becomes important. The linear regime of the detector is confined to the following two conditions: First, only a small fraction of the full well capacity is used. Second, the photon generated current dominates the dark current of the det , tor diode.

\section{OVERVIEW OF INFRARED ARRAYS AT ESO}

In Table 1 the detector arrays tested at ESO and installed in various instruments are listed. The first array introduced at ESO was a linear $1 \times 32 \mathrm{InSb}$ array bonded to a Reticon multiplexer with a pixel size of $200 \mu \mathrm{m}$. The limitation of the Reticon readout is due to the fact that all pixels must share a common video line. The read noise was reduced to $1200 \mathrm{e} \mathrm{rms} \mathrm{by}$ a special weighted double correlated clamp (Finger et al. 1987a). This detector was installed in the cryogenic infrared spectrometer IRSPEC in 1985 and was in service until 1991.

The first two-dimensional array tested at ESO was a $64 \times 64 \mathrm{Hg}_{1-\mathrm{x}} \mathrm{Cd}_{\mathrm{x}} \mathrm{Te}$ array read out by a CCD. The cutoff wavelength of the detector is $4.2 \mu \mathrm{m}$ but can be set between $2.5 \mu \mathrm{m}$ and $10 \mathrm{~mm}$ by varying the composition $\mathrm{x}$ of the $\mathrm{Hg}_{1-\mathrm{x}} \mathrm{Cd}_{\mathrm{x}} \mathrm{Te}$ alloy. The detectors consist of cylindrically shaped $\mathrm{p}-\mathrm{n}$ junctions which are connected to a buried channel CCD by a special loophole interconnect technology (Finger et al. 1987b). The input stage of the CCD consists of a direct injection circuit. At low flux levels the charge injection efficiency drops, since the input impedance of the direct injection circuit is inversely proportional to the current. Another problem is the threshold uniformity of the injection circuit which requires very uniform doping concentration of the buried channel CCD. The MCT CCD was installed in the infrared array camera IRAC1 in 1988.

Advances in the large scale integration of silicon made it possible to place a source follower in the unit cell of each detector. Multiplexers having one source follower per detector (SFD) have become the generally accepted way of read out. A 64 x 64 InSb array was tested in the lab and the famous $58 \times 62$ InSb SBRC array (Fowler et al. 1987) replaced the linear array in IRSPEC in 1991. The possibility of tuning the band gap of the alloy $\mathrm{Hg}_{1-\mathrm{x}} \mathrm{Cd}_{\mathrm{x}} \mathrm{Te}(\mathrm{MCT})$ to the energy of a photon of $\lambda=2.5 \mathrm{~mm}$ allows the subdivision of the $\lambda=$ one to five $\mathrm{mm}$ spectral range in to the non-thermal infrared shortward of $\lambda=2.5 \mathrm{~mm}$ which is covered by MCT 
TABLE 1

Infrared Arrays at ESO

\begin{tabular}{lllllllllll}
\hline \hline Company & Device & Format & $\lambda_{c}$ & Instrument & $\begin{array}{l}\text { Read } \\
\text { out }\end{array}$ & $\begin{array}{l}\text { Pixel } \\
\text { pitch }\end{array}$ & Well & Noise & QE \\
CINCINNATI & InSb & $1 \times 32$ & 5 & IRSPEC & $\begin{array}{l}\text { Reti- } \\
\text { con }\end{array}$ & 200 & $2.410^{7}$ & 1200 & 0.85 \\
MULLARD & MCT & $32 \times 32,64 \times 64$ & 4.2 & IRAC1 & CCD & 48 & $3.110^{6}$ & 200 & 0.28 \\
CINCINNATI & InSb & $64 \times 64$ & 5 & LAB & SFD & 100 & $210^{6}$ & 180 & 0.75 \\
SBRC & InSb & $58 \times 62$ & 5 & IRSPEC & SFD & 75 & $3.210^{5}$ & 200 & 0.8 \\
ROCKWELL & MCT & $256 \times 256$ & 2.5 & IRAC2A & SFD & 40 & $210^{5}$ & 25 & 0.55 \\
CINCINNATI & InSb & $256 \times 256$ & 5 & LAB & SFD & 30 & $8.410^{5}$ & $?$ & 0.8 \\
SBRC & InSb & $256 \times 256$ & 5 & IRAC2B & SFD & 30 & $510^{5}$ & 120 & 0.65 \\
ROCKWELL & MCT & $1024 \times 1024$ & 2.5 & ISAAC & SFD & 18.5 & $610^{4}$ & $<10$ & 0.6 \\
SBRC & InSb & $1024 \times 1024$ & 5 & ISAAC & SFD & 27 & $310^{5}$ & 25 & 0.8 \\
LIR & Si:Ga & $64 \times 64$ & 18 & TIMMI & SFD & 100 & $310^{7}$ & 2500 & 0.25 \\
& & $128 \times 192$ & & & & 75 & & & 0.3 \\
\hline
\end{tabular}

detectors. Longward of $\lambda=2.5 \mathrm{~mm}$, in the regime of the thermal infrared, the $1-5 \mathrm{~mm}$ response of InSb detectors is utilized. The big step both in performance and array format came with the NICMOS3 $256 \times 256 \mathrm{Hg}_{1-\mathrm{x}} \mathrm{Cd}_{\mathrm{x}} \mathrm{Te}$ array having a cutoff wavelength of $2.5 \mu \mathrm{m}$. This array has been producing science in the large infrared camera IRAC2A since 1992 (Moorwood et al. 1992). At present two high well InSb $256 \times 256$ arrays are being tested at ESO. The Cincinnati array uses an extra capacity of $0.17 \mathrm{pF}$ at the gate of the source follower which augments the detector capacitance of $0.1 \mathrm{pF}$, resulting in an integrating node capacity of $0.27 \mathrm{pF}$ (Blessinger et al. 1993). The SBRC array uses the standard CRC-463 multiplexer and will be discussed in more detail below.

Both ROCKWELL and SBRC are developing 1024 x 1024 arrays which will be complementary with respect to wavelength response, readout speed and noise performance. With growing array format there is a general tendency towards smaller pixels and lower pixel capacity. The $\lambda=8-13 \mu \mathrm{m}$ region is served by a $64 \times 64 \mathrm{Si}: \mathrm{Ga}$ array manufactured by LETI/LIR. In 1993 this array was installed in TIMMI, the ESO $10 \mu \mathrm{m}$ camera built by the Service d'Astrophysique at Saclay. A new $128 \times 192$ successor of this array is under development (Lucas et al. 1994). 


\section{NICMOS3 MCT $256 \times 256$ ARRAY}

The NICMOS3 array is an excellent device in most respects and has been evaluated extensively. Results are discussed in more detail elsewhere (Finger et al. 1993). The characteristics of NICMOS3 are given in Table 2. The array has a very low dark current of 0.4 $\mathrm{e}^{-} / \mathrm{sec}$ due to its short cutoff wavelength of $\lambda_{\mathrm{c}}=2.5 \mathrm{~mm}$ and an excellent noise performance of $20 \mathrm{e}^{-}$rms.

\section{TABLE 2}

NICMOS3 MCT 256 x 256 Array Characteristics

\begin{tabular}{ll}
\hline \hline Detector & $\mathrm{Hg}_{1-\mathrm{x}} \mathrm{Cd}_{\mathrm{x}} \mathrm{Te} \mathrm{LPE}$ on $\mathrm{Al}_{2} \mathrm{O}_{3}$ substrate buffered by CdTe (PACE 1) \\
\hline Wavelength range & $1-2.5 \mu \mathrm{m}$ \\
Number of pixels & $256 \times 256$ \\
Pixel Pitch & $40 \mu \mathrm{m}$ \\
Read-out & $\mathrm{CMOS}$ shift registers organized in 4 independent quadrants, SFD unit cell \\
Output amplifiers & $4(1$ per quadrant) \\
Package & 68 pin LCC \\
Full Well & $210^{5} \mathrm{e}^{-}$ \\
Noise & $20 \mathrm{e}^{-} \mathrm{rms}$ with multiple sampling \\
QE & $52 \%$ at $2.2 \mu \mathrm{m}, 29 \%$ at $1.25 \mu \mathrm{m}$ \\
Darkcurrent & $<0.4 \mathrm{e}^{-} / \mathrm{sec}$ \\
Operating Temperature & $70 \mathrm{~K}$ \\
Problems & reset anomaly, amplifier glow, persistence \\
Status & fully tested, operating in IRAC2 since May 1992 \\
\hline
\end{tabular}

However, the array suffers from some problems relevant to low flux applications. One problem is the persistence effect. If the array is exposed to ambient temperature K-band radiation a subsequent dark exposure displays a dark-current of $9 \mathrm{e}^{-} / \mathrm{sec}$, which decays exponentially and decreases to $2.7 \mathrm{e}^{-} / \mathrm{sec}$ after $700 \mathrm{sec}$. Subelectron darkcurrent can only be achieved after the detector has been kept dark for many hours. The reset anomaly is another problem with which to cope. After the reset strobe has been applied to the detector diodes to recharge the integrating node capacitance, the integration ramp is strongly nonlinear during the first $100 \mathrm{msec}$. For this reason the first nondestructive readout in the multiple nondestructive read scheme has to be discarded. Only the linear part of the integration ramp should be used. Multiple nondestructive sampling is adversely affected by the luminescence of the multiplexer which is strongest in the corners of the array close to the output amplifiers. In the center of the array, where this effect is smallest, a $300 \mathrm{sec}$ dark exposure with 656 nondestructive 
readouts gives a signal of 7582 electrons. The signal consists of the following components: 3400 electrons may be assigned to the amplifier glow, 3900 electrons are due to the shift registers and 270 electrons are due to the diode dark current. The photon shot noise generated by the multiplexer glow limits the reduction of read noise by multiple sampling to $20 \mathrm{e}^{-} \mathrm{rms}$.

\section{HIGH WELL CAPACITY SBRC $256 \times 256$ InSb ARRAY}

Since the one to five micron wavelength region covers both the non-thermal and the thermal infrared, the detector arrays used for multimode instruments at large 8-m class telescopes have to cope with flux levels varying from less than one photon/sec for high resolution spectroscopy to $10^{8}$ photons/sec for $\mathrm{M}$ band imaging. If all instrument modes must be served by a single detector, a trade-off between read noise and full well has to be considered. Small pixel capacities result in excellent noise figures but require prohibitively high read speeds for broad band imaging in the thermal infrared. At an eight-m telescope broad band imaging in L of a 46"x 46" field with the standard SBRC $256 \times 256 \mathrm{InSb}$ array requires that one pixel is read out in less than $435 \mathrm{nsec}$ if all four quadrants are read in parallel.

Two possibilities have been explored to increase the capacity of the integrating node. An extra capacity can be added to the gate of the source follower in the unit cell of the multiplexer (Blessinger et al. 1993). This requires a special multiplexer design. Alternatively, the pixel capacity can be increased by increasing the doping concentration of the detector diode. The capacity of the pn junction is proportional to the square root of the doping concentration $\mathrm{N}_{\text {doping }}$ as shown in equation 4:

$$
C(V)=A_{\text {Diode }} \sqrt{\frac{\varepsilon_{\text {InSb }}{ }^{N} \text { doping }^{e}}{2\left(V_{\text {builtin }}+V\right)}}+C_{\text {Gate }}
$$

SBRC has developed a special high doped $256 \times 256$ InSb array hybridized to a CRC 463 multiplexer which is compared to the low doped standard InSb array in Table 3. The integrating capacitance is approximately $0.19 \mathrm{pF}$, much higher than the $0.06 \mathrm{pF}$ for the standard low-doped arrays. With a $400 \mathrm{mV}$ bias, the well capacity becomes $4.810^{5}$ electrons. Typical noise on the high-doped arrays is 100 electrons rms, compared with 55 electrons for low-doped arrays. Preliminary dark current measurements which may be distorted by thermal background photons due to imperfect shielding gave $15 \mathrm{e} / \mathrm{sec}$ at $350 \mathrm{mV}$ reverse bias. The cosmetic quality of the array is good. It has 270 bad pixels 170 of which are clustered at the edge of the array. The expected readout time for one pixel is increased to more than one $\mu \mathrm{sec}$ for broad band imaging in $\mathrm{L}$, which is feasible with state of the art electronics. The quantum efficiency specified in Table 3 was measured by the manufacturer.

\section{SPECIAL READOUT TECHNIQUES}

To reduce the read noise we implemented the nondestructive multiple sampling scheme. After applying the reset strobe the array is read out many times, but without resetting the integrating node capacitance to the external bias voltage, which is illustrated in Fig. 2. During the stare time the integration ramp is sampled nondestructively as often as the bandwidth of the data acquisition system allows. The images read nondestructively are used to update the 
TABLE 3

Comparison of Standard and High Doped SBRC 256 x 256 InSb Array Detector

\begin{tabular}{|c|c|c|c|c|c|}
\hline Detector & C & Well & Noise & QE & pixel time \\
\hline & [pF] & [e] & [e rms] & [\%] & [nsec] \\
\hline standard & 0.06 & $1.8510^{5}$ & 55 & 80 & 435 \\
\hline high Well & 0.19 & $4.810^{5}$ & 100 & 65 & 1377 \\
\hline
\end{tabular}

error equations of a regressional fit to the integration ramp. The read noise $\Delta \mathrm{Nrmsn}$ for $\mathrm{n}$ nondestructive readouts per detector integration is given by equation (5), $\Delta \mathrm{Nrms}$ being the read noise of double correlated sampling:

$$
\Delta N r m s_{n}=\Delta N r m s \sqrt{\frac{6(n-1)}{n(n+1)}} \approx \Delta N r m s \sqrt{\frac{6}{n}} \text { for } \mathrm{n} \gg>1
$$

In principle nondestructive readout can reduce the read noise to negligible amounts by increasing the detector integration time and the number of possible readouts. In practice other limitations arise, e.g. the NICMOS detector becomes shot noise limited by the luminescence of the multiplexer for integrations longer than three seconds.

\section{MULTIPLE NONDESTRUCTIVE SAMPLING}

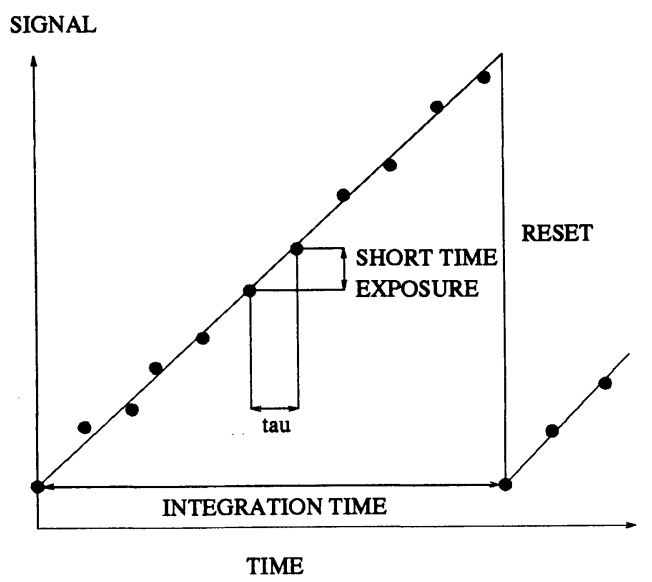

Fig. 2. Illustration of multiple nondestructive sampling readout and on-chip tracking algorithm. The difference of two nondestructive readouts corresponds to a short time exposure which can be recentered to compensate image motion due to atmospheric seeing. 
Ground based astronomical observations suffer from seeing effects which result from distortions of the plane wavefront of a star image by atmospheric turbulence (Roddier et al. 1992). Within the nondestructive readout scheme a powerful technique of image sharpening can be implemented. This technique is called on-chip tracking and allows correction of image motion in real time which is equivalent to correction of first order wavefront perturbations (Finger et al. 1993, Finger et al. 1994a, b). The information required to compensate the image motion is contained in the difference of two consecutive readouts. The difference is equivalent to a short time exposure taken during the time interval between two readouts. Positional information obtained from a guide star within the field seen by the array is used to shift the whole data array representing non-destructive readouts. The shifted differences are used to reconstruct the integration ramp. A threshold set for the peak intensity of the guide star can be defined to discard moments of bad seeing.

On chip tracking has the same effect as a tip tilt correction by an active optical element (Close and McCarthty 1994) - but without the extra complexity of adding such a device. A comparison of on chip tracking with simple shift and add using destructive readout and double correlated sampling shows that the readout speed can be increased by a factor of three without any hardware modifications. Furthermore, under-sampled images profit from the noise advantage of multiple sampling.

The tracking mode has been tested at a 2.2-m telescope in the $\mathrm{K}$ band with the infrared array camera IRAC2 equipped with the NICMOS3 array. Under good seeing conditions the spectral bandpass is well tuned to the telescope diameter to perform first order wavefront corrections since the ratio of the telescope diameter $D$ to the Fried coherence parameter $r_{0}$ is expected to be $\mathrm{D} / \mathrm{r}_{0} \sim 3$ at $\lambda=2.2 \mathrm{~mm}$ (Roddier et al. 1992). Fig. 3 shows a subframe of a three second exposure containing the pre-main-sequence binary star S CrA. The left image is untracked while the right image is tracked. The data are untreated raw data. The pixel scale is 0.14 arcseconds which means that the diameter of the airy disk is sampled by 3.7 pixels. The detector was read out nondestructively every $100 \mu \mathrm{sec}$. Tracking improves the Strehl ratio by a factor of 2.7 and the binary star is well resolved.

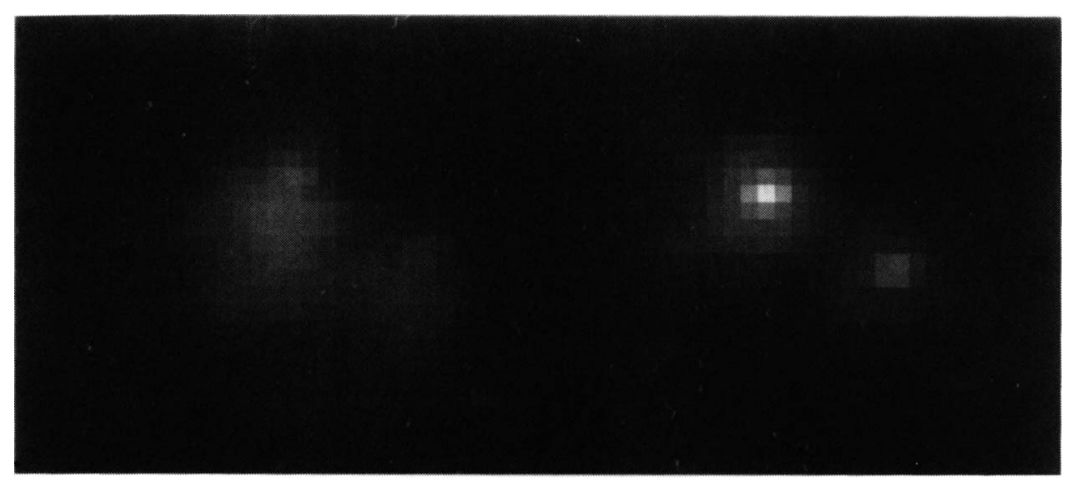

Fig. 3. K' image of the pre main sequence binary star S CrA. Left: untracked. Right: tracked. Integration time three sec. Pixel scale: 0.14 arcseconds. 


\section{FUTURE LARGE FORMAT ARRAYS}

Currently two projects are under development with the aim of producing an intrinsic megapixel IR focal plane array. One of them is the HAWAII project - a collaboration between the University of Hawaii and Rockwell to develop an MCT array for low background applications in the $1-2.5 \mu \mathrm{m}$ spectral range; the other is the ALADDIN project, a collaboration between NOAO and SBRC for the 1 to $5 \mu \mathrm{m}$ range. The characteristics of both developments are summarized in Table 4. Both detectors are organized in four quadrants. The ALLADDIN detector has eight parallel video outputs per quadrant to allow the parallel processing necessary for the high read speeds required for $5 \mu \mathrm{m}$ imaging.

The key issues to be solved for the large format of megapixel arrays are the yield of the silicon readout chip and the thermal mismatch between the detector substrate and the silicon multiplexer. Both project groups have demonstrated that reasonable yields for the multiplexer production can be obtained. The HAWAII detector has already been hybridized and has been used to image successfully the SL9/Jupiter collision event. The thermal mismatch problem is still under investigation. Efforts are being made to improve the array stability with thermal cycling. Amplifier glow, reset anomaly and persistence effect are promised by the manufacturers to be eliminated or at least greatly reduced. The Infrared Spectrometer and Array Camera ISAAC built for the VLT could be upgraded with these arrays when they become available (Moorwood 1993).

\section{INFRARED ARRAY CONTROL ELECTRONICS - IRACE}

The advent of the next generation megapixel infrared arrays initiated the development of a new modular high speed data acquisition system to read out a wide variety of infrared detectors in all possible read-out modes. The design is mainly driven by the requirement to read out the 32 parallel video channels of the SBRC 1024 x $1024 \mathrm{InSb}$ detector in $50 \mathrm{msec}$. The performance goal is to achieve shot noise limited operation in the wavelength region $\lambda=1$ to $5 \mu \mathrm{m}$. The specifications for IRACE depend on the flux levels to be expected in a multimode instrument, which may vary over many orders of magnitude depending on the observing mode. Broad band imaging in the $\mathrm{L}$ band has to cope with the thermal emission of telescope, which may contribute as much as $2.310^{6} \mathrm{e}^{-} / \mathrm{sec} /$ pixel for a field of $1.3^{\prime} \times 1.3^{\prime}$ at an eight-m telescope assuming the pixel size of the SBRC 1024 x $1024 \mathrm{InSb}$ array. To avoid saturation by the thermal background the detector has to be read out in less than $65 \mathrm{msec}$. If 32 channels are operated in parallel, the time available to read out one pixel is $2 \mathrm{msec}$ resulting in an overall data rate of $513 \mathrm{Mbit} / \mathrm{sec}$. Since broad band M filters used in astronomy are not well matched to the atmospheric window, only narrow band filters centered in the best part of the atmospheric window will be used with little loss in sensitivity and flux levels similar to broad band $\mathrm{L}$.

In contrast, in the high resolution spectroscopic mode for a spectral resolution of $\mathrm{R}=$ 15000 the flux of zodiacal light between $\mathrm{OH}$ lines at $\lambda=1.665 \mu \mathrm{m}$ is as low as $0.04 \mathrm{e} / \mathrm{sec} / \mathrm{pixel}$. The detector darkcurrent is claimed by the manufacturers to be as low as $0.1 \mathrm{e} / \mathrm{sec}$. Integration times will be ten minutes and the detector can be read out with low read speed and low electronic bandwidth to achieve read noise values below $25 \mathrm{e}^{-} \mathrm{rms}$ corresponding to a noise voltage of $80 \mathrm{mV}$ at the detector output. 
TABLE 4

Characteristics of Large Format 1024 x 1024 Infrared Arrays as Given by the Manufacturer

\begin{tabular}{|c|c|c|}
\hline Detector & ROCKWELL 1024x1024 MCT & SBRC $1024 \times 1024 \mathrm{InSb}$ \\
\hline Nickname & HAWAII & ALADDIN \\
\hline Detector material & $\begin{array}{l}\mathrm{HgCdTe} \mathrm{LPE} \text { on } \mathrm{Al}_{2} \mathrm{O}_{3} \text { substrate buffered } \\
\text { by CdTe (PACE } 1)\end{array}$ & Thinned, backside illuminated InSb \\
\hline Wavelength range & $1-2.5 \mu \mathrm{m}$ & $0.9-5.2 \mu \mathrm{m}$ \\
\hline Number of pixels & $1024 \times 1024$ & $1024 \times 1024$ \\
\hline Pixel Pitch & $18.5 \mu \mathrm{m}$ & $27 \mu \mathrm{m}$ \\
\hline Read-out & CMOS, 4 quadrants, Reset only by rows & CMOS, 4 quadrants, Reset only by \\
\hline Output amplifiers & 4 (1 per quadrant), & 32 (8 per quadrant) \\
\hline Package & 84 pin LCC & 124 pin LCC \\
\hline Frame Rate & $1.2 \mathrm{~Hz}$ & $20 \mathrm{~Hz}$ \\
\hline Full Well & $110^{5} \mathrm{e}$ & $310^{5} \mathrm{e}^{-}$ \\
\hline Noise & $8.6 \mathrm{e} \mathrm{rms}$ & $25 \mathrm{e}^{-} \mathrm{rms}$ \\
\hline $\mathrm{QE}$ & $>50 \%$ & $>80 \%$ \\
\hline Darkcurrent & $<0.1 \mathrm{e} / \mathrm{sec}$ & $<0.1 \mathrm{e} / \mathrm{sec}$ \\
\hline Operating Temperature & $>50 \mathrm{~K}$ & $35 \mathrm{~K}$ \\
\hline
\end{tabular}

The most important design feature is the clear and uncompromising galvanic separation of the analog part of the acquisition system contained in the detector front end and the digital part contained in the number cruncher. They are connected by an ultra fast 500 Mbit fiber link.

Inside the cryostat next to the detector there are filters and antistatic protection circuits for clocks and dc bias voltages. A flexible manganin board interfaces the detector to a warm preamplifier mounted closely to the detector. The preamplifier generates a symmetrical analog signal which is sent to the detector front end (DFE) outside the dewar. In the DFE the sequencer generates the timing pattern required to scan the detector multiplexer. It consists of a size four TRAM (T805) and loads the clock pattern into a $32 \times 2048$ bit fifo buffer to guarantee quartz stable timing of 32 clocks. The minimum time state is 50 nsec with a minimum average change of the clock pattern every 200 nsec. The TTL clocks are converted 
to analog clocks by fast CMOS switches (HI-201HS) in the clock and dc bias driver module which also generates the dc voltages. The video processor module has true differential inputs and bessel filters for 32 video channels. They can be sampled in parallel with 500 nsec time resolution and 16 bit accuracy ADC's (ANALOGIC ADC4322). The ultra fast one Gbaud fiber link (Siemens V23806-A5-C1) transmits the digitized data to the number cruncher and prevents ground loops from introducing noise into the analog signal chain.

The number cruncher is realized by a transputer network. Multiple nondestructive readout with linear regression of the integration ramp requires one multiplication and two additions for running Gaussian sums within 2 msec for one megapixel read out by 32 parallel video channels. The transputer employed for this task is the $20 \mathrm{Mhz}$ Inmos T9000. If this transputer is not available in time a backup solution with digital signal processors (25 Mhz Texas TMS320C40) is envisaged. The advantage of the modular transputer concept is the possibility to eventually perform more data processing in real time by additional and more powerful transputers.

\section{CONCLUSIONS}

During the last decade photovoltaic IR arrays have been subject to dramatic improvements. Both the array format has increased from $1 \times 32$ to $256 \times 256$ and the pixel performance has improved. Infrared focal plane technology is still in its revolutionary phase of development. Megapixel arrays will be available within one year. The pixel performance is approaching fundamental sensitivity limits. Novel sampling techniques for noise reduction and image sharpening have been developed.

Large format arrays are needed for the next generation of instruments built for eight-m telescopes. Better image quality obtained by adaptive optics requires sampling to higher spatial frequencies. The large fields for which these instruments are designed increase the demand for large array formats. VLT instruments developed for ESO (ISAAC, CONICA) will profit enormously from the advent of megapixel arrays.

\section{REFERENCES}

Blessinger, M. A., Apgar, G. W., Biggs, J. D., Louderback, M. L. and Niblack, A. N. 1993 Proc. SPIE 1946, 55

Close, L. M. and McCarthy, D. W. 1994 PASP 106, 77

Finger, G., Biereichel, P., Meyer, M. and Moorwood, A. F. M. 1993 Proc. SPIE 1946, 134

Finger, G., Biereichel, P., Meyer, M. and Moorwood, A. F. M. 1994 Infrared Astronomy with Arrays: The next Generation, I. S. McLean, ed., UCLA, p. 345

Finger, G., Biereichel, P., van Dijsseldonk, A., Meyer, M. and Moorwood, A. F. M. 1994 Proc. SPIE 2198, 763

Finger, G., Meyer, M. and Moorwood, A. F. M. 1987a Proceedings of the Workshop on Ground-based Observations with Infrared Array Detectors, University of Hawaii, Institute for Astronomy, Honolulu, p. 60

Finger, G., Meyer, M. and Moorwood, A. F. M. 1987b Proc. SPIE 865, 94

Fowler, A. M., Probst, R. G., Britt, J. P., Joyce, P. R. and Gillet, F. C. 1987 Optical Engineering 26, 3, 232

Lucas, C., Pantigny, P., Alloin, D., Cesarsky, C., Kaeufl, H. U. and Monin, J. L. 1994 Infrared Astronomy with Arrays: The Next Generation, I. S. McLean, ed., UCLA, p. 425 
Moorwood, A. F. M. 1993 Proc. SPIE 1946, 461

Moorwood, A. F. M., Finger, G., Biereichel, P., Delabre, B., Van Dijsseldonk, A., Huster, G., Lizon, J. -L., Meyer, M., Gemperlein, H. and Moneti, A. 1992 The Messenger 69, 61

Roddier, F., Northcott, M. and Graves, J. E. 1992 PASP 103, 131 